\title{
A MODEL FOR MODE I FAILURE OF LAMINATED COMPOSITES
}

\author{
S. J. Song ${ }^{1}$ \\ Anthony M. Waas ${ }^{2}$ \\ The University of Michigan \\ Ann Arbor, Michigan
}

\begin{abstract}
A new model has been developed to predict mode I failure of laminated composites. Instead of using an energy release rate concept based on Linear Elastic Fracture Mechanics (LEFM), a nonlinear elastic foundation model was employed to simulate cohesive interlaminar fracture of the composite. The materials experiencing stresses due to the presence of the crack was replaced by a nonlinear elastic foundation with its own special properties. Unidirectionally laminated DCB type specimens were used in this study to model the mode I failure. A finite element code was developed to solve the beam on a nonlinear elastic foundation model. The current model reproduced load versus displacement curves of laboratory fracture tests very closely. The current model has the potential capability of being extended to predict the evolution of planar crack growth for 2-D crack problems without recourse to an explicit crack growth criterion.
\end{abstract}

\section{INTRODUCTION}

The use of composite materials in recent times has been escalating rapidly in a broad spectrum of industry as these materials turn out to be an excellent engineering alternative to traditional materials due to outstanding mechanical and chemical properties such as high flexibility, light weight, excellent fatigue strength and corrosion resistance etc. However composite structures have revealed weaknesses in directions perpendicular to their lamination planes. For example, debonding between two distinct materials or delamination in laminated fibrous composites is a major concern. Therefore the issues related to fracture in these new bi-material based structures has been and continues to receive much attention.

The same concepts that were used to analyze homogeneous isotropic materials were naturally adopted to this new area with some modifications [1]. However the complexity inherent in the crack problems associated with these materials inhibits its use from being an easy

1. Graduate Research Assistant

2. Asst. Professor, Aerospace Engineering, Member AIAA. Copyright c 1993 by Anthony M. Waas. Published by the American Institute of Aeronautics and Astronautics, Inc. with permission. task or makes the solution of the corresponding fracture mechanics problem almost impossible for some combinations of materials [1]. Many researchers have made their contributions to enhancing the understanding of crack problems in laminated composites by doing experiments [2-10]. Chai [2-4] performed experiments to study the effect of bond thickness on fracture toughness using adhesive joints. He used aluminum beams as adherends and various kinds of polymers as adhesives with different adhesive thicknesses. From these experiments, he showed that the critical energy release rate is not a true material constant. He also explained that the reason there is so much scatter in this critical value for the same material is that the fracture toughness is critically dependent, additionally, on the morphology revealed on cracked surfaces of fracture specimens.

It appears that linear elastic fracture mechanics may not always deliver the proper tools to handle crack problems in layered materials. Motivated by this, we have developed a new fracture model; namely a nonlinear elastic foundation model with properties to characterize the foundation that are dependent on critical quantities of the constituents such as maximum strength, Young's modulus etc. DCB type specimens were used in this study to model mode I delamination of a laminated composite. The model is called a beam on a nonlinear elastic (spring) foundation in which the cracking material is represented by continuous springs. The concept of a spring representation has been mentioned and studied by many previous workers. At the early stages of fracture mechanics development, Elliot [11] used a continuum solution for a cracked body under uniform tension, where he studied two semi-infinite blocks that attract each other with interatomic forces. The interactions were schematically represented by springs. Later Goodier and Kanninen [11] extended Elliot's model by introducing nonlinear springs as well as linear springs that attach pairs of atoms. But this model had size limitations due to considerations based on distances between neighboring atoms. Later, in the 1980 's, Kanninen used a beam on a linear spring foundation model to calculate the energy release rate of mode I fracture of a DCB [12]. He also used the same model to study fast fractures in DCB type specimens [13]. Similar concepts along with the finite element technique can be found in several places[14-16]. Nonlinear elastic foundation models to study crack propagations 
without an energy release rate concept have been attempted by some researchers [17-19]. However a linear foundation is used and its constitutive property is not clearly defined $[17,18]$, or the cracking material carries load even after reaching its maximum strength [19].

In this study, a new nonlinear elastic foundation model with its own special properties have been developed. The characterization of the current model is illustrated in section 2. Development of a finite element to solve the beam on nonlinear foundation model is explained in section 3. An experimental study is described in section 4. Finally sections 5 and 6 present results and conclusions, respectively.

\section{A MECHANICAL MODEL FOR CRACK PROPAGATION}

Fig. 1.a shows a laminated composite DCB fracture specimen and Fig. 1.b is the corresponding beam on a nonlinear elastic foundation model for mode I failure.

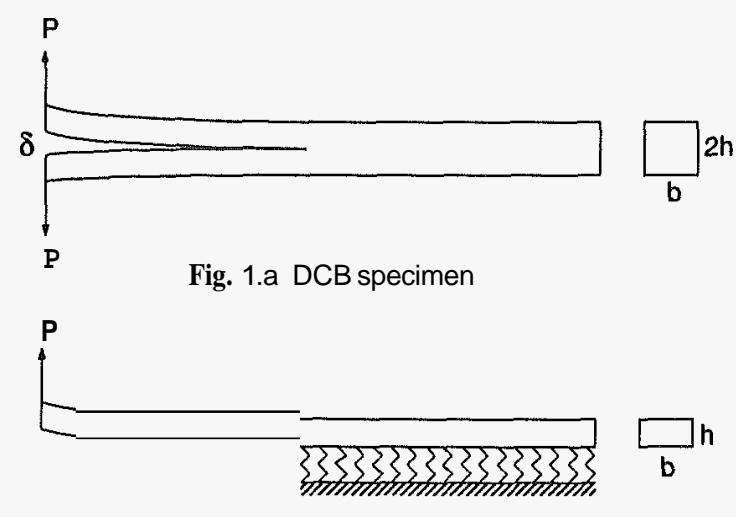

Fig. 1.b Beam on nonlinear foundation model

The foundation is a Winkler type spring foundation. There is no friction between any pair of adjacent springs. The DCB considered in this study is uni-directionally laminated with the fiber direction along the beam axis. The crack is at the center of resin rich matrix material.

This model has two parts and each part plays an individual role. One is the beam part and the other is the spring or elastic foundation part. The beam is responsible for accounting for the bending deformation caused by external loading. The spring part is responsible for the transverse deformation at the crack tip which will eventually cause fracture. As the external loading increases, the springs continue to deform until the first spring at the crack tip reaches its maximum endurable strength. At the maximum strength, the material becomes unstable and fracture occurs right away without additional energy absorption during breaking of the material. This phenomenon can be explained using the following analogy. Consider a cylindrical bar made of nonlinear elastic material subjected to uniaxial tensile loading. The bar is of a finite length and infinitesimal cross-sectional area. Suppose we model the bar as a series of non-linear springs having slightly different constitutive laws for each spring. The maximum strength the system can reach equals the maximum strength of the weakest spring. As the weakest spring passes its maximum strength and becomes unstable (the descending portion of the stressstrain curve), the other springs are still ascending up the stress vs strain curve, approaching their own maxima. At this stage the system becomes unstable because the weakest spring cannot attain a higher force while the others can. Once the system reaches this point, without further global loading or extension, the weakest spring pulls apart permanently. No more energy absorption from external loading occurs at this point; a redistribution of strain energy occurs because the breaking spring (the deformation is localized at this spring) experiences an increased strain energy while a corresponding decrease in strain energy occurs in the other springs, thus making the total strain energy constant.

In the present spring model, it is assumed that the energy from the transverse normal strain is much larger than the energy from shear and axial strain caused by the crack under mode I failure. Therefore shear and axial terms were not accounted for.

\subsection{Characterization of Spring Foundations}

In this model, the spring is assumed to deform uniformly through the thickness. In other words, the spring has constant strain throughout the length. The constitutive relation for this spring is assumed to be

$$
\sigma=E_{1} \varepsilon e^{-E_{2} \varepsilon}
$$

where $E$, and $E$, are positive constants to be determined. This form is an approximation to the inter-atomic force law and is depicted in Fig. 2. We need two conditions to solve for $E$, and $E$. The first condition is that the initial slope of the stress versus strain curve is equal to Young's modulus of the matrix material, $E_{\text {,, }}$. The second condition is that energy the spring can absorb up to the maximum strength is equal to the surface energy (half the fracture energy) determined from a single laboratory fracture test for a given laminate. After applying these two conditions we obtain 


$$
\begin{aligned}
& E,=E_{m} \\
& E_{2}=\left(\frac{t E_{m}}{\gamma}\left(1-\frac{2}{e}\right)\right)^{\frac{1}{2}}
\end{aligned}
$$

where $t$ is the spring length and $\gamma$ is the surface energy. The spring length is taken to be half the resin layer thickness of the laminate with an assumption that the plies above the resin rich layer is much stiffer than the resin layer so that the energy absorbed in the plies above the resin layer can be neglected. The resulting force versus displacement relation for the current spring foundation can be obtained by substituting (EQ 2) into (EQ 1) along with $\mathrm{E}=w / t$. This final equation will be used in the development of the finite element in section 3 .

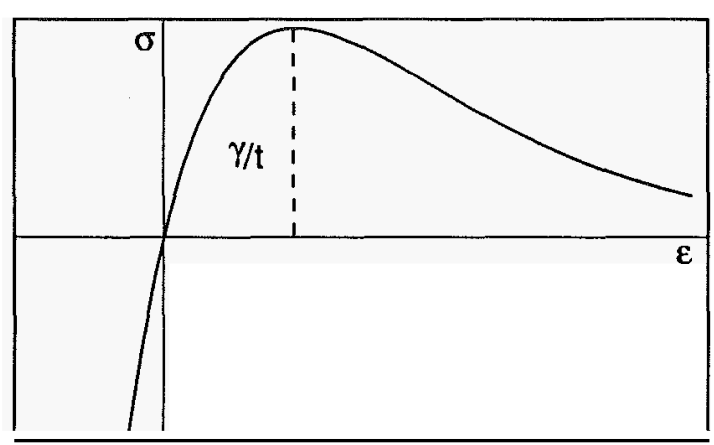

Fig. 2 Constitutive relation for current model

The failure criterion for the spring foundation can be based on either its maximum strength or its critical strain. The critical strain was chosen in this study. It follows that the crack is assumed to be propagated if the first spring at the crack tip has reached its critical strain, the strain at which the slope of the stress versus strain curve becomes zero (Fig. 2).

\section{FINITE ELEMENT IMPLEMENTATION}

A finite element code was developed to solve the beam on nonlinear foundation model in an incremental way. Bernoulli-Euler beam theory was used to model the beam deformation. A multilinear spring constitutive law, depicted in Fig. 3, was used to handle the general nonlinearity of the cracking material (spring). The stationary potential energy theorem was employed to develop the element stiffness matrices and equivalent nodal loads. Details are as follows.

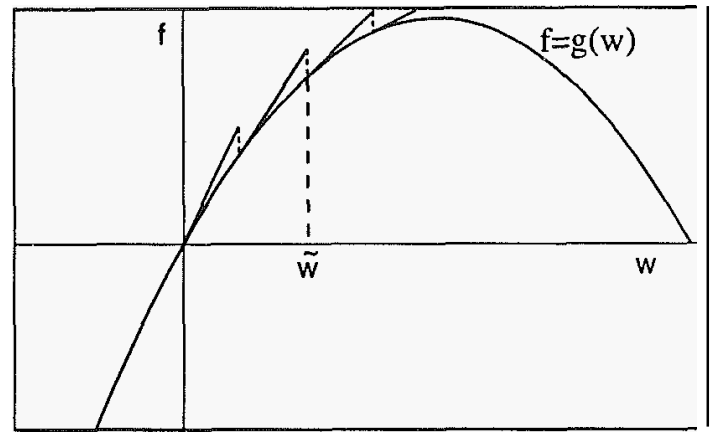

Fig. 3 Multilinearspring model

The nonlinear spring foundation has a force versus displacement relation of the form

$$
f=g(w)
$$

where $f$ is a force with units of stress and $w$ is the transverse deflection of the beam centroid. The beam itself has no transverse strain. So the beam and spring foundation have the same amount of deflections w. Now assume that the spring has deformed up to $\tilde{w}$ in an incremental way. During the proceeding increment the spring deforms to a new state $w$. Therefore the force versus displacement relation during this increment ( $\tilde{w}$ to $w$ ) can be written as,

$$
f=k_{1} w+k_{2}
$$

where $k$, and $k$, are given by,

$$
\begin{aligned}
& k,=g^{\prime}(\tilde{w}) \\
& k_{2}=g(\tilde{w})-\tilde{w} g^{\prime}(\tilde{w})
\end{aligned}
$$

The potential energy of the linearized system is

$$
\Pi=U_{\text {beam }}+{ }_{\text {spring }}-W_{e}
$$

where $U$ denotes the strain energy and $W_{e}$ is the external work potential. Each of these are as follows

$$
\begin{aligned}
& U_{\text {beam }}=\frac{1}{2} E I \int_{0}^{L}\left(w^{\prime \prime}\right)^{2} d x \\
& U_{\text {spring }}=b \int_{0}^{L}\left[\tilde{U}+\int_{\tilde{w}}^{w}\left(k_{1} w+k_{2}\right) d w\right] d x \\
& W_{e}=w_{1} F_{1}+\theta_{1} M_{1}+w_{2} F_{2}+\theta_{2} M_{2}
\end{aligned}
$$


where $\tilde{U}$ is a strain energy the element has absorbed up to $\tilde{w}$. Now the deflection $\boldsymbol{w}$ can be approximated using beam shape functions.

$$
w(x)=w_{1} N_{1}+\theta_{1} N_{2}+w_{2} N_{3}+\theta_{2} N_{4}
$$

where $N$, through $N_{4}$ are Bernoulli-Euler beam shape functions. After substituting the approximate deflection $w(x)$ into (EQ 5), the energy equation is differentiated with respect to nodal displacements. The stationary potential energy theorem can be applied for equilibrium,

$$
\begin{aligned}
\delta \Pi & =\frac{\mathrm{an}}{\partial w_{1}} \delta w_{1}+\frac{\mathrm{an}}{\partial \theta_{1}} \delta \theta_{1}+\frac{\mathrm{an}}{\partial w_{2}} \delta w_{2}+\frac{\mathrm{an}}{\partial \theta_{2}} \delta \theta_{2} \\
& =0
\end{aligned}
$$

This leads to the following element matrix equation.

$$
\left[K_{i j}^{\text {beam }}+K_{i j}^{\text {spring }}\right]\left[\begin{array}{l}
w_{1} \\
\theta_{1} \\
w_{2} \\
\theta_{2}
\end{array}\right]=\left[\begin{array}{c}
F_{1} \\
M_{1} \\
F_{2} \\
M_{2}
\end{array}\right]+\left[\begin{array}{l}
R_{i} \\
]
\end{array}\right.
$$

where

$$
\begin{aligned}
& K_{i j}^{\text {beam }}=E I \int_{0}^{L} N_{i}^{\prime \prime} N_{j}^{\prime \prime} d x \\
& K_{i j}^{\text {spring }}=\underset{0}{b \int_{0}^{L} k_{1} N_{i} N_{j} d x} \\
& R_{i}=-b \int_{0}^{L} k_{2} N_{i} d x
\end{aligned}
$$

Here, the indices $i, j$ range from 1 to $\mathbf{4}$ and $R$, are the equivalent nodal loads created from the nonlinearity of the spring foundation. Note that $k_{1}$ and $k$, are functions of $\tilde{w}(\mathbf{x})$. There are two ways of integrating $K_{i j}^{\text {spring }}$ and $R_{i}$ matrices. One method is using numerical integration and the other option is through exact integration after simplification. The latter method is chosen in this study in which $\tilde{\boldsymbol{w}}(x)$ can be approximated to have a single average value within one finite element. In this manner $k_{1}$ and $k$, can be taken outside the integral sign. This is possible only if the element size is very small for those elements having high gradient in deflection. Thus, a dis- advantage of this method is the large total number of finite elements to be used. However, a major advantage is that it avoids numerical integrations all throughout the domain of integration, which usually takes a considerable amount of CPU time. Our problem has a small region of high gradient in deflection; only near the crack tip. This fact made the latter method a better choice for our problem.

A mesh was generated in such a manner that element numbering is dense near the crack tip and sparse away from the crack tip. The resulting global matrix equation was solved using Gauss elimination with pivoting. The numbering of global matrix was done diagonally so as to reduce the size of computer memory, hence reducing the CPU time also. The loading on the DCB was simulated by incrementing the displacement at the loading point. During the crack propagation phase, each time the spring at the crack tip reaches its breaking condition, the program calculates how much the crack has propagated and regenerates a new mesh according to the new crack length until the crack has propagated to the final crack length. This numerical process acquires another convergence problem in addition to convergence problems associated with the finite element method itself. This convergence problem was solved by reducing the increment size of the loading until convergence was found to occur.

\section{EXPERIMENTAL STUDY}

A mode I fracture test was performed to validate the current model. The DCB type specimen was made by cutting E7T1-2BI/G40-800 unidirectional 48 ply laminated composite plate obtained from BP Chemical Corp. Loading was done slowly with a speed of $0.4 \mathrm{~mm}$ per minute on a screw driven Riehele testing frame, simulating static conditions. Special grips were made to minimize any unnecessary moment and shear arising from grips. The crack opening displacement (6 in Fig. 1) was measured via an LVDT and the corresponding load was measured via a $200 \mathrm{lbf}$ load cell. The instantaneous location of the crack tip was marked on one side of the beam with the aid of a magnifying glass. The beam specimen was prepainted white to make the crack tips more visible. Both readings and time were acquired on an in-house data acquisition system. Fig. 4 depicts the pulling force, $P$, versus crack opening displacement, 6 , for the beam specimen. After the test, the side of cracked specimen was examined using a microscope along the crack line. It was found that the failure was fully interlaminar (not intralaminar) and cohesive. The thickness of the resin rich layer was taken to be the average of several measurements through a high magnification microscope. Three 


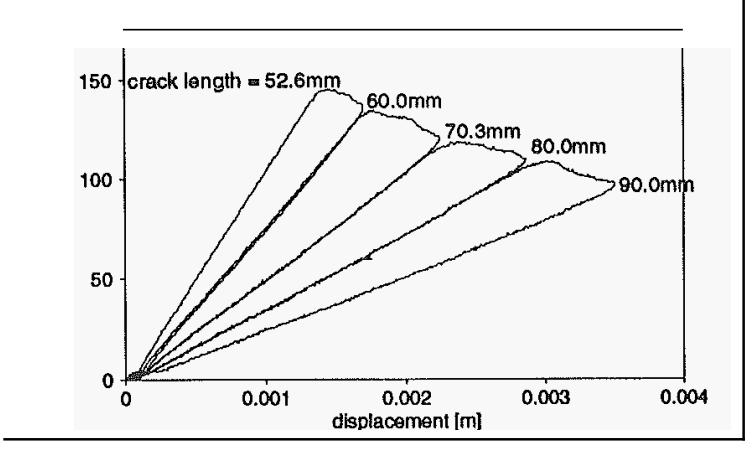

Fig. 4 Fracture test result of E7T1-2BI/G40-800 DCB

point bending test was done to obtain the bending stiffness of the beam, which was used as an input data for the current model.

\section{RESULTS}

Fig. 5.a is the output of current model after simulating E7T1-2BI/G40-800 DCB specimen with the resin layer thickness of $35 \mu \mathrm{m}$. The output is compared with experimental result in Fig. 5.b. The current model has reproduced the experiment very well. The surface energies

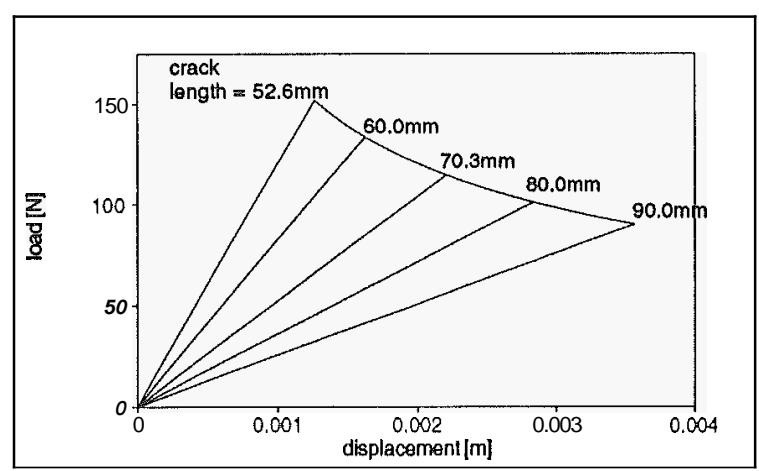

Fig.5.a Simulation output

obtained from the experiment were averaged to be used in this model. The average fracture energy calculated from the experiment and that from the current model matched very closely with an error of less than $1 \%$. The slopes of the loading and unloading curves were found to match more closely as the crack length became large compared with the beam thickness. Bernoulli-Euler beam theory was used in this model, and its predictions become more accurate with an aspect ratio of bigger than 20 for unidirectional composite beams. It was found that the slopes of the unloading curves in Fig. 5.b for both experiment and simulation are somewhat lower than they

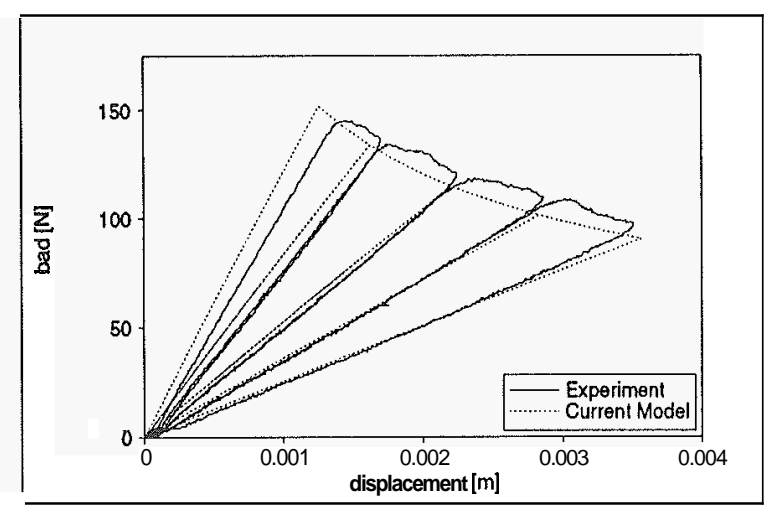

Fig. 5.b Comparison between experiment and simulatior

would be if the cantilever beam approach was used. This indicates that the material at the crack tip is displacing up and rotating as the cracking material is being deformed due to the external loading. Thus, the 'slope' of the crack flange is never zero at the crack tip. This is in contrast to a cantilever approximation to the crack flanges. This nonzero slope was experimentally observed by Parvin and Knauss [20].

\section{CONCLUDING REMARKS}

The current model uses fracture energy from a single laboratory fracture test as an important condition to characterize the spring property. It is insensitive to details at the crack tip, and therefore is easy to characterize and use for virtually any kind of mode I fracture specimen. This model reproduced the fracture tests of unidirectionally laminated composite beam specimens very closely.

The advantage of the current model is that microscopically behaving cracking material which experiences high gradients in stress and large strain is separated out from the global structure which has relatively low gradients in stress and strain. Another important strength is that the current model can be utilized to predict the evolution of planar crack propagation with any initial planform shape of crack geometry, whereas fracture mechanics concepts based on energy release rates cannot in a straightforward manner (except for simple geometries) be extended to achieve the same tasks (for example the growth of delaminations of general shapes in impacted laminates; in this case the 'beam' theory is replaced by 'plate' theory and the formulation is in a 2D setting).

Our current application of the model can be improved by using 2-D elasticity elements instead of beam elements as the latter revealed weaknesses for the case of short crack lengths. A spring model for mode II crack propagation is being developed using similar concepts. A 
mixed mode failure model, then, can emerge after combining both models in an appropriate manner.

\section{ACKNOWLEDGEMENTS}

During the course of the investigation the authors received financial support through ONR contract N-0001491-5-1705. We are grateful for this support and appreciate the interest of Dr. Y.D.S. Rajapakse, the program monitor. We would also like to thank Dr. A. Babayan from BP Chemical Company, LA, for the donation of the specimens.

\section{REFERENCES}

1. Hutchinson, J.W. and Suo, Z. "Mixed mode cracking in layered materials", Advances in Applied Mechanics, Vol. 28, Academic Press. 1991

2. Chai, H. "The characterization of Mode I delamination failure in nonwoven multidirectional laminates", Composites, Vol. 15, No. 4, Oct. 1984

3. Chai, H. "Bond thickness effect in adhesive joints and its significance for Mode I interlaminar fracture of composites", Composite Materials (Seventh Conference), ASTM, 1986

4. Chai, H. "On the correlation between the Mode I failure of adhesive joints and laminated composites", Engineering Fracture Mechanics, Vol. 24, No. 3, pp. 413-431, 1986

5. Williams, J. G. "On the calculation of energy release rates for cracked laminates", International Journal of Fracture 36: 101-119(1988)

6. Williams, J. G., Hashemi, S. and Kinloch, A. J. "The analysis of interlaminar fracture in uniaxial fibre-polymer composites", Proc. R. Soc. Lond. A427, 173-199 (1990)

7. Suo, Z. "Failure of brittle adhesivejoints", Appl Mech Rev vol 43, no 5, Part 2, May 1990

8. Suo, Z., Fan, B. and Bao, G. "Delamination R-curve phenomena due to damage", Journal of Mechanics and Physics of Solids, Revised in Dec. 11, 1990

9. Suo, Z. and Hutchinson, J. W. "Interface crack between two elastic layers", International Journal of Fracture 43: 1-18, 1990

10. Ungsuwarungsri, T. and Knauss, W. G. "A nonlinear analysis of an equilibrium craze: Part I, II", Journal of Applied Mechanics. Vol. 55, March 1988

11. Kanninen, M. F. “Advanced Fracture Mechanics", Oxford Engineering Science Series 15. 1985

12. Kanninen, M. F. "An augmented double cantilever beam model for studying crack propagation and arrest", International Journal of Fracture, Vol. 9, No. 1, March 1973

13. Kanninen, M. F. "A dynamic analysis of unstable crack propagation and arrest in the DCB test specimen", International Journal of Fracture. Vol. 10. No. 3. September 1974

14. Wang, S. S."An analysis of the crack tip stress field in DCB adhesive fracture specimens", International Journal of Fracture. Vol. 14. No. 1.February 1978

15. Roelfstra, P. E. "Simulation of failure in computer generated structures", Fracture Toughness and Fracture Energy. SENDAI 12-14October 1988, pp 313-324

16. Hoff, R., Rubin, C. A. and Hahn, G. T. "A new finite element technique for modelling stable crack growth", EngineeringFracture Mechanics. Vol. 23. No. 1. pp. 105118. 1986

17.Leonardi, A., Maceri, F. and Sacco,E. "Debonding of bimodular plates", International Series of Numerical Mathematics, Vol. 101. 1991,pp113-128.

18. Shilkrut, D. "Unilateral contact between a geometrically non-linear plate and elastic foundation", Proceedings of the Second Meeting on Unilateral Problems in Structural Analysis, Proceedings, June 17-20, 1985.

19. Ungsuwarungsri, T. and Knauss, W. G. "The role of damage-softened material behavior in the fracture of composites and adhesives", SM 85-4-1, Revised and shortened ver. of Ph.D. Thesis, Grad. Aero. Lab. Cal. Tech. (1986)

20. Parvin, M. and Knauss, W. G. "Damage induced constitutive response of a thermoplastic related to composites and adhesive bonding", International Journal of Fracture 42: pp57-72, 1990 